\title{
Does a Leaking O-Corner Save the Square?
}

\author{
Pieter A.M. Seuren
}

\begin{abstract}
It has been known at least since Abelard (12th century) that the classic Square of Opposition suffers from so-called undue existential import (UEI) in that this system of predicate logic collapses when the class denoted by the restrictor predicate is empty. It is usually thought that this mistake was made by Aristotle himself, but it has now become clear that this is not so: Aristotle did not have the Conversions but only one-way entailments, which 'saves' the Square. The error of UEI was introduced by his later commentators, especially Apuleius and Boethius. Abelard restored Aristotle's original logic. After Abelard, some 14th- and 15th-century philosophers (mainly Buridan and Ockham) meant to save the Square by declaring the $\mathbf{O}$-corner true when the restrictor class is empty. This 'leaking O-corner analysis', or LOCA, was taken up again around 1950 by some American philosopher-logicians, who now have a fairly large following. LOCA does indeed save the Square from logical disaster, but modern analysis shows that this makes it impossible to give a uniform semantic definition of the quantifiers, which thus become ambiguousan intolerable state of affairs in logic. Klima (Ars Artium, Essays in Philosophical Semantics, Medieval and Modern, Institute of Philosophy, Hungarian Academy of Sciences, Budapest, 1988) and Parsons (in Zalta (ed.), The Stanford Encyclopedia of Philosophy, http://plato.standford.edu/entries/square/, 2006; Logica Univers. 2:3-11, 2008) have tried to circumvent this problem by introducing a 'zero' element into the ontology, standing for non-existing entities and yielding falsity when used for variable substitution. LOCA, both without and with the zero element, is critically discussed and rejected on internal logical and external ontological grounds.
\end{abstract}

Keywords Abelard · Aristotle · Boethius · Buridan · Existential import · Null element · O-Corner · Predicate negation · Restricted quantification · Square of Opposition

Mathematics Subject Classification Primary 03B65 · Secondary 03B50

\section{Letting the 0-Corner Leak}

Some 14th-century nominalist philosophers, such as Buridan and Ockham and a bevy of 15th-century philosophers (Ashworth [1]), and then again some 20th-century American philosophers, notably Moody [4], Thompson [10], Klima [3] and Parsons [5, 6], were struck by the fact that the classic Square of Opposition (henceforth the Boethian Square or just the Square) is saved from the logical defect of undue existential import (UEI) if it proves possible to assign existential import only to the affirmative left-hand side of 
the Square but not to the negative right-hand side. This way, existential import depends on what is traditionally called the quality of the sentence type and not on its quantity: when the restrictor predicate $\mathrm{F}$ has a null extension $(\llbracket \mathrm{F} \rrbracket=\varnothing),{ }^{1}$ falsity is assigned to A- and I-type sentences but truth to $\mathbf{A}^{*}$ - and $\mathbf{I}^{*}$-type sentences. ${ }^{2}$ This proposal is often characterized as the proposal to let the $\mathbf{0}$-corner 'leak', in that the $\mathbf{O}$-corner ( $\boldsymbol{I}^{*}$ or $\neg \mathbf{A}$ ) drops its existential import. In fact, however, it is not just the $\mathbf{O}$-corner that is made to 'leak' but also the E-corner $\left(\mathbf{A}^{*}\right.$ or $\left.\neg \mathbf{l}\right)$ of the Square. To avoid any possible negative connotations, the less suggestive acronym LOCA will be used instead of the full phrase 'leaking O-corner analysis'.

Many modern philosophers of this school, including Parsons, and to a lesser extent also Moody, claim that LOCA is the way the Square was interpreted by Aristotle and throughout the Middle Ages. This claim, however, is unsustainable. Aristotle proposed a logic, later elaborated by Abelard (Seuren, [8]: Ch. 5), without the defect of UEI. This Aristotelian-Abelardian system does not contain the classic Conversions $\left(\mathbf{A}^{*} \equiv \neg \mathbf{I}\right.$ and $\mathbf{I}^{*} \equiv \neg \mathbf{A}$ ) but only one-way entailments (A entails $\neg \mathbf{I}$ and $\mathbf{I}^{*}$ entails $\neg \mathbf{A}$ but not vice versa; see Fig. 3). So there is no question of a 'leaking' O-corner there. As regards the proposals made by Buridan and Ockham, they were not only tentative and barely elaborated but also highly controversial in their day. ${ }^{3}$ The extreme scarcity of passages in the 14thcentury philosophical literature actually attesting LOCA shows the tenuousness of this claim as far as the 14th century is concerned (Seuren, [8]: 158-168). ${ }^{4}$ Ashworth ([1]: 144) shows that throughout the 15 th century, up till the early 16th century, there was a group of philosophers who saw a parallel between singular sentences with an empty subject term, such as (1a) and the corresponding existentially quantified sentence (1b), which were both considered false in the absence of any chimera.

(1) a. This chimera is a mammal.

b. Some chimera is a mammal.

c. This chimera is not a mammal.

d. Some chimera is not a mammal.

e. No chimera is a mammal.

\footnotetext{
${ }^{1}$ The standard double bracket notation is used for predicate extensions: $\llbracket \mathrm{F} \rrbracket$ stands for all elements in the universe of entities ENT for which the predicate $F$ delivers truth.

${ }^{2}$ The eight sentence types used are $\mathbf{A}$ ( $A l l F$ is $G$ ), I (Some $F$ is $G$ ), their external negations $\neg \mathbf{A}$ and $\neg \mathbf{I}$, their internal negations $\mathbf{A}^{*}$ and $\mathrm{I}^{*}\left(\mathrm{All} / \mathrm{Some} \mathrm{F}\right.$ is not $\mathrm{G}$ ), and their external and internal negations $\neg \mathbf{A}^{*}$ and $\neg^{*}$ (Seuren, [8]: 31-37). The old E-corner is thus split up into $\neg \mathbf{I}$ and $\mathbf{A}^{*}$ and the old $\mathbf{O}$-corner into $\neg \mathbf{A}$ and $\mathbf{I}^{*}$. $\neg \mathbf{I}$ and $\mathbf{A}^{*}$ and $\neg \mathbf{A}$ and $\mathbf{I}^{*}$ can thus be distinguished in systems where the members of each pair are not equivalent.

3“'Ockham's Summa Logicae (The Logic Handbook), written ca. 1323, is a manifesto masquerading as a textbook" (King, [2]: 243).

${ }^{4}$ It is difficult to find a 14th-century text actually stating LOCA, instead of merely playing with the idea. One such text is Ockham, Summa Logicae, beginning of Chap. II.3 (http://individual.utoronto.ca/ pking/resources/Ockham?Summa_logicae.txt):
}

$<$ Q $>$ uandoque sufficit quod subiectum indefinitae vel particularis negativae pro nullo supponat. $(<S>$ ometimes it suffices for the truth of a negative indefinite or particular proposition that the subject refers to nothing.)

For ample commentary and a full text of the passage in question, see Seuren ([8]: 163-166). 

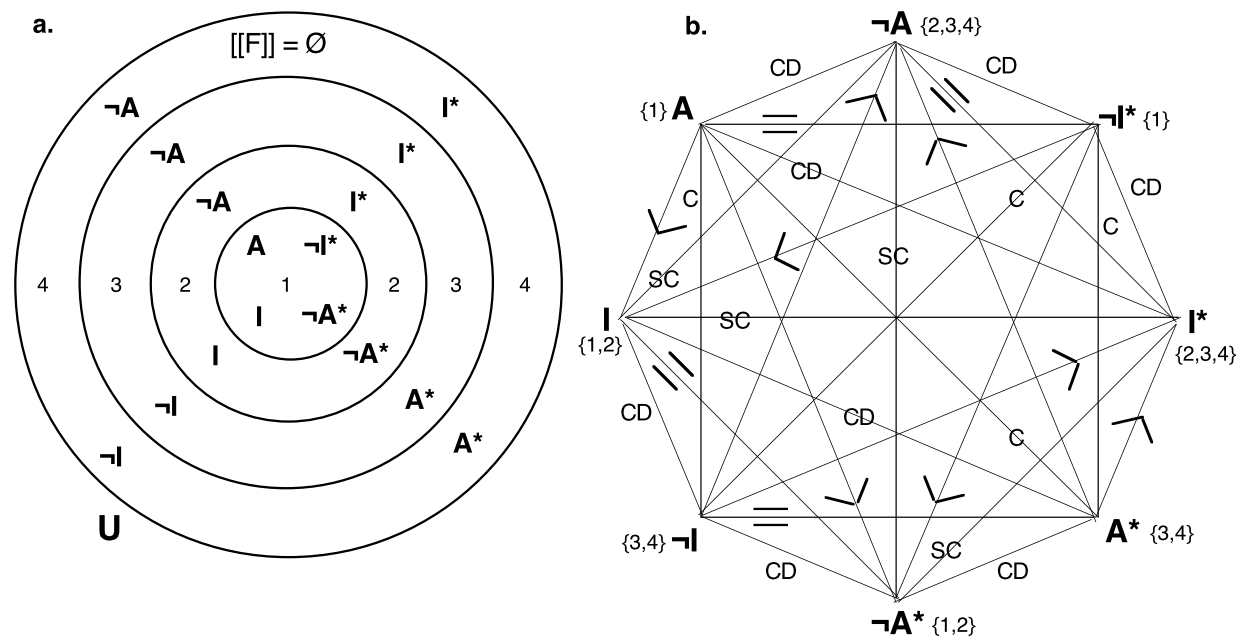

Fig. 1 The predicate logic in terms of LOCA

They reasoned that, just as (1c), the negation of (1a), is true, the apparent quantificational analog (1d) should, therefore, also be true. What these late medieval philosophers overlooked, however, is that whereas (1c) is the negation of (1a), (1d) is not the negation of (1b). The negation of (1b) is (1e), which destroys the analogy. After c. 1500 nothing is heard of this misguided analysis any more, until it was revived in America around 1950.

Apart from this historical claim, however, it is true that, on the face of it, LOCA saves the Square. We then get a logic that preserves all logical relations of the Square. That this is so is shown by Fig. 1, which shows the LOCA-based VS-model on the left and the corresponding octagon on the right, ${ }^{5}$ which is identical with the octagon corresponding to the Square, shown in Fig. 2 (where space 4 in Fig. 2a has been left blank owing to UEI). This makes LOCA look interesting. The question is: is this tenable?

One immediate reason for not considering LOCA tenable is that it fails at least one test for logical soundness, the MODULO-*-PRINCIPLE. This principle says that the logic of quantifiers is independent of whether the restrictor or F-predicate and/or the matrix or G-predicate are or are not negated. That is, the semantics of the quantifiers must be the same whether or not the F- or G-predicate are negated. A violation of this principle means that the logic at hand is no longer definable in terms of the meanings of its operators. Thus, if, in cases where $\llbracket F \rrbracket=\varnothing$, All $F$ is $G$ and Some $F$ is $G$ are declared false but All $F$ is not- $G$ and Some $F$ is not-G true, it follows that the semantic definitions of the universal and the existential quantifiers vary depending on whether a positive or a negative predicate

\footnotetext{
${ }^{5}$ For explicit exposés of Valuation Space (VS) modeling and corresponding polygonal representations of logical systems, see Seuren ([8]: 46-50; [9]: Ch. 6). Intuitively, the notions are clear enough. A VS model splits up the universe $\mathbf{U}$ of possible situations into classes of situations in which each sentence type of a logical system is true. The valuation space /S/ of a sentence $\mathrm{S}$ is the set of situations in which $\mathrm{S}$ is true. For example, in Fig. $2, / \mathbf{A} /=\{1\}, / / /=\{1,2\}$, etc. A polygonal representation is a polygon with as many vertices as there are sentence types in the system (without vacuous repetitions of negations). Lines between vertices stand for logical relations (equivalence or $=$, entailment or $>$, contradictoriness $\mathrm{Cr}$, contrariety or C, subcontrariety or SC).
} 

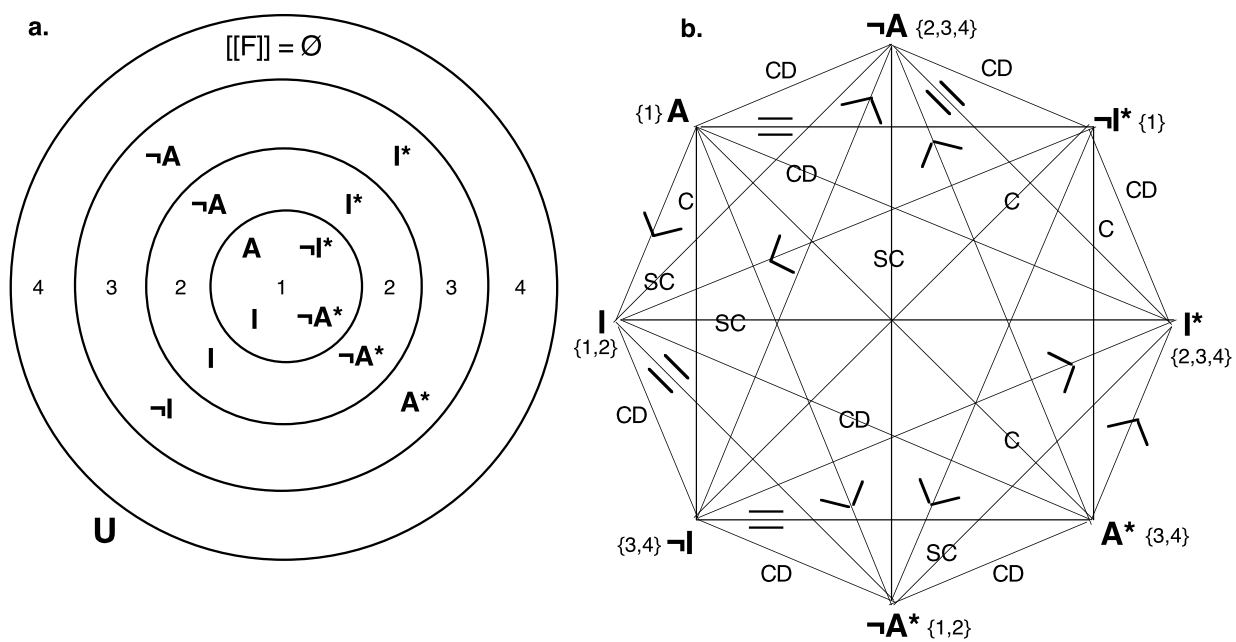

Fig. 2 The VS-model and the octagonal representation of the classic Square

is chosen. This makes it impossible to provide a uniform definition for the quantifiers concerned that is valid for all situations and for all values for the matrix G-predicate. It is shown below that this is borne out dramatically by Parsons' attempt at saving the Square.

No distinction can be made between the compound but single negative predicate not- $\mathrm{P}$ - with the so-called 'predicate negation' — and not $\mathrm{P}$ (without the hyphen), for any predicate $P$, as the only negation defined in the system is the logical negation $\neg$ and a 'predicate negation', as in not- $P$, is simply defined by saying that $\llbracket$ not- $P \rrbracket$ is $\llbracket \neg(P(x) \rrbracket$, the complement of $\llbracket P \rrbracket$, or $\llbracket \bar{P} \rrbracket$, in the universe of all entities ENT. The hyphen in not-P thus cannot make any logical difference, given the formal means at our disposal within the framework of a bivalent extensional logic. Yet in LOCA, Some F is not G is considered true but Some $F$ is not-G (with the single predicate not-G) false, when $\llbracket F \rrbracket=\varnothing$, which means that the hyphen does make a logical difference there. ${ }^{6}$

The question is important because, in LOCA, the distinction between not-P and not $P$ has stridently counterintuitive consequences, as it implies that Some mermaids are unmarried is false in this world but Some mermaids are not married is true. Moreover, a sentence like Some mermaid is not a mermaid would have to be considered true but Some mermaid is a mermaid false. This is, in fact, sufficient to reject out of hand this solution for the purpose of establishing a natural predicate logic. Yet it is worth our while to have a further look.

\footnotetext{
${ }^{6}$ When the logic turns presuppositional, the hyphen will make a logical difference. As Aristotle already observed, the only generalization that can be made for a predicate $P$ and its negative counterpart not- $P$ (or unP, or whatever morphological or lexical means the language has available for creating negative predicates) is that they make for contrary sentences, which cannot both be true, but can both be false. A relation of contradiction between sentences made with $\mathrm{P}$ and not- $\mathrm{P}$ is restricted to those predicates that are nongradable and have no presuppositional preconditions, such as existent and nonexistent. In presuppositional logic, this means that the predicate-internal negation can only be presupposition-preserving. But the not in not $\mathrm{P}$, without the hyphen, can be the radical presupposition-canceling not that yields truth in cases of presupposition failure.
} 

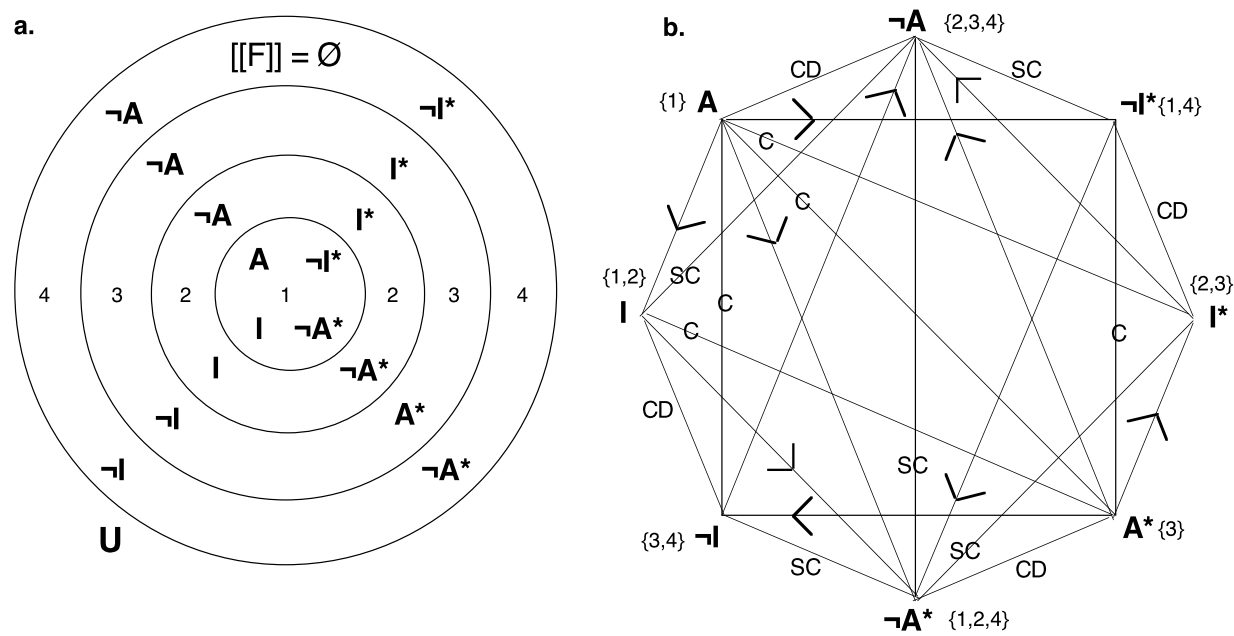

Fig. 3 The VS-model and the octagon for AAPL

Moody ([4]: 51-52) admits the counterintuitiveness of the proposal, adding that, therefore, the O-corner should be read as 'not all' rather than as 'some not', thereby implicitly, but, it seems, unknowingly, giving up the Boethian Square with its Conversions for the Abelardian Square with its one-way entailments (see Fig. 3). Thompson ([10]: 253) says, implicitly confessing to the misconception that logic is predestined for all eternity instead of being defined by the meanings of its operators:

Even if we agree with the new defenders of Aristotle that the decision which leads to the modern analysis is repugnant to ordinary speech, we can still argue that this is more desirable than a decision repugnant to logical analysis itself.

Parsons declares ([6]: 5):

\begin{abstract}
What is important is that the logical notation be coherent and useful. If it does not perfectly match the usage of ordinary language, that is not on its own important for a system of logic. Indeed, if you are sure that ordinary language universal affirmatives should be false when their subject term is empty, then you may represent that fact by translating them into modern logical notation adding a conjunct. Instead of symbolizing 'Every $\mathrm{A}$ is $\mathrm{B}$ ' by ' $\forall \mathrm{x}(\mathrm{Ax} \rightarrow \mathrm{Bx})$ ', symbolize it as $' \exists x A x \& \forall x(A x \rightarrow B x)$ '.
\end{abstract}

Here again, both authors are, apparently, unaware that Aristotle did not have the Conversions but only one-way entailments, which, far from being 'repugnant to logical analysis itself', stays well within the range of natural intuitions and keeps the logic sound as well as free from undue existential import. Then, while Thompson uncritically takes it for granted that saving natural logical intuitions leads to logical disaster, Parsons overlooks the fact that adding a nonnull condition to the semantic definition of $\forall$ yields a different logic, Aristotelian-Abelardian predicate logic (AAPL), shown in Fig. 3.

But apart from the intuitively rebarbative character of the proposal, let us have a look at the more technical details. Klima ([3]: 18-2) and Parsons [6] are the only authors who actually present a logical system incorporating LOCA, rather than just stating that I*-type sentences should be considered true when $\llbracket F \rrbracket=\varnothing$. Klima resorts to an old medieval device (revived, it seems, by the late 19th-century British philosopher Hugh MacColl, 
referred to in Russell, [7]: 491) of introducing a null or zero element $\boldsymbol{\varnothing}$ standing for any nonexistent entity. Some late medieval philosophers (followed, apparently, by MacColl) used $\varnothing$ to solve what is now known as the problem of reference failure. They posited that the definite description the unicorn in a sentence like The unicorn is running refers to the null element $\varnothing$ when, as in the actual world, there are no unicorns. This made it possible to assign falsity to this sentence and thus to escape the predicament caused by reference failure, while maintaining strict bivalence.

Klima and Parsons, interpreting ø model-theoretically, posit that zero-element substitution in the range $\llbracket P \rrbracket$ of an extensional predicate $P$ (no account is taken of intensional predicates) is admissible when $\llbracket P \rrbracket=\varnothing$, but leads not to truth but to falsity. This is at least remarkable because, by definition, substitution restricted to the extension $\llbracket P \rrbracket$ of a predicate $\mathrm{P}$ simply lists all members of $\llbracket \mathrm{P} \rrbracket$ and thus always yields truth.

Around 1950, the machinery of variable substitution had become widely known and was seen as the center-piece of the semantics for the Russellian language of predicate logic, where it seemed to work well (provided ENT $\neq \varnothing$ ). At that time, the British philosopher Peter Geach was making headlines opposing the Russellian system of unrestricted quantification, so called because in this system every variable ranges over the whole of ENT. Geach proposed the theory of restricted quantification, where variables rotate not over the whole of ENT but only over $\llbracket F \rrbracket$, the extension of the restrictor predicate. This, however, landed Geach and his followers in the predicament that in cases where $\llbracket F \rrbracket=\varnothing$ no substitution is possible for the matrix predicate $G(x)$, so that the truth value of propositions like $\exists x: F(x) \mid[G(x)]$ or $\forall x: F(x) \mid[G(x)]$ must remain undecided when $\llbracket F \rrbracket=\varnothing .^{7}$ Geach never solved this problem, but Klima saw that, with the help of medieval $\varnothing$, these propositions turn out false — good enough, it would seem, for a respectable predicate logic.

Klima follows Geach's system of restricted quantification. This means that the variable in a formula like $\forall x: F(x) \mid[G(x)]$, or 'for all $x$ such that $x$ is an $F, G(x)$ ', is allowed to rotate only over $\llbracket F \rrbracket .^{8}$ As has been said, substitution of $\boldsymbol{\sigma}$ is considered legitimate whenever $\llbracket \mathrm{F} \rrbracket=\varnothing$, but it yields falsity.

Now consider the $I^{*}$-type Some $\mathrm{F}$ is not $\mathrm{G}$, formulated, in the language of restricted quantification, as (2):

(2) $\exists x: F(x) \mid \neg[G(x)]$

Suppose $\llbracket F \rrbracket=\varnothing$, which, in Klima's analysis, means that $\varnothing$ is the only admissible substitution. Substitution of $\varnothing$ for $x$ makes $G(x)$ false and thus $\neg[G(x)]$ true. Therefore, one might feel justified to conclude that there is one substitution that produces truth for the

\footnotetext{
${ }^{7} \mathrm{~A}$ formula like $\forall \mathrm{x}: F(x) \mid[G(x)]$ is read as 'for all $x$ such that $x$ is an $F, G(x)$ ', and $\exists x: F(x) \mid[G(x)]$ as 'for some $x$ such that $x$ is an $F, G(x)$ '. In this restricted quantification notation, the part between the colon and the upright stroke is the range of the restricted quantifier $(\forall x$ or $\exists x)$ and the part after the upright stroke is the argument. It should be noted that the use of a first-order logical language in no way implies the use of the a particular first-order logical system. When the Russellian language is used here, it is because it has been found satisfactory for the present purpose. The language of restricted quantification is used when it is found useful for that purpose. A given formal logical language can be used with different semantic interpretations according to the logical system under discussion. The important thing is that the set-theoretical basis underlying any logical system is made explicit in a convenient, consistent and unambiguous way.

${ }^{8}$ Klima's own notation is slightly different, but amounts to the same. I skip a discussion of Klima's elaborate formal and semantic description of his system, as it easily reduces to restricted quantification.
} 
function $\neg[\mathrm{G}(\mathrm{x})]$. And since the satisfaction of the existential quantifier $\exists$ requires that there be at least one truth-yielding substitution for $\mathrm{x}$, the substitution of $\boldsymbol{\varnothing}$ for $\mathrm{x}$ makes (2) true despite (or perhaps because of) the fact that $\llbracket F \rrbracket=\varnothing$.

The same result follows for $\mathbf{A}^{*}$-type sentences formulated as:

(3) $\forall \mathrm{x}: \mathrm{F}(\mathrm{x}) \mid \neg[\mathrm{G}(\mathrm{x})]$

Again, $\neg[\mathrm{G}(\boldsymbol{\varnothing})]$ (with $\varnothing$ for $\mathrm{x}$ ) is true, and since $\boldsymbol{\varnothing}$ is the only admissible substitution, all admissible substitutions produce truth, which makes (3) true. Truth thus results vacuously for $\mathbf{I}$-type and $\mathbf{A} *$-type sentences when $\llbracket F \rrbracket=\emptyset$.

Although this looks good at first sight, there are serious problems connected with the zero element $\boldsymbol{\emptyset}$. First, if $\boldsymbol{\varnothing}$ makes for falsity under any (extensional) predicate, how about the predicate be the zero element? When I say "ø is the zero element," then this should be false despite the fact that $\varnothing$ is the zero element! But apart from this paradox, a set-theoretic problem arises when it is asked whether $\boldsymbol{\varnothing}$ does or does not belong to the extension of a predicate $P$. If it does, why does it produce falsity? If it does not, why is it an admissible substitution within the range of $\llbracket P \rrbracket$ ? From the point of view of ontology, $ø$ is likewise highly problematic. No ontology will come up with such an element on independent grounds. It is merely an artifice, introduced for the sake of getting logic out of trouble without sacrificing the Bivalence Principle.

Critical readers might well ask why the null element should be considered so objectionable while the null set is fully accepted and treated with respect. The answer is that the null set is solidly anchored in modern set theory and makes for straightforward and sound systems of predicate logic, such as modern standard predicate logic or AAPL, whereas the null element would upset set theory no end and prevents a uniform semantic definition of the quantifiers in all logical languages except the language of restricted quantification. Yet, the objectors may observe, ontologically speaking, the null set is every bit as questionable as the null element: no ontologist would come up with such a notion on independent grounds. And here we can only agree. In fact, when the logic is made intensional, allowing, as it should, for virtual or intensional entities, the null set is written out of the script altogether. The assumption into the ontology of virtual entities, along with the screening off of presupposition failure by means of a special radical negation, obviates the need for both the null set and the null element. But this aspect cannot be addressed in the present paper (for a full discussion see Seuren, [8]: Ch. 10).

Parsons [6] tries to achieve the same as Klima, but in a different and rather far-fetched formalism, which, however, again boils down to restricted quantification. For Parsons, all categorical sentences center around the identity formula $\mathrm{x}=\mathrm{y}$. Both variables must be bound by a quantifier (EVERY or SOME) for a proposition (sentence) to arise. ${ }^{9}$ The negation (NOT) can be inserted in front of the whole sentence, between quantifiers, and in front of $\mathrm{x}=\mathrm{y}$. The substitution mechanism is again of the restricted kind: variable ranges are restricted to a given predicate extension. When either $\mathrm{x}$ or $\mathrm{y}$ in $\mathrm{x}=\mathrm{y}$ fails to find a value in their range, $\mathrm{x}=\mathrm{y}$ is reckoned to be false. Parsons uses the phrase "when nothing is assigned to $\mathbf{x}$ or to $y$," but this cannot be taken literally, because when an element $\mathbf{e}$ is substituted for $y$ and nothing is substituted for $x$, all one has is a formula ' $x=e^{\text {', which }}$ is a sentential function (or predicate) and thus cannot have a truth value. It is hard to see

\footnotetext{
${ }^{9}$ Parsons has a third quantifier NO, but, as far as I can make out, this NO is equivalent with NOT SOME, in his system.
} 
how this obstacle can be removed without positing a zero element, with all the problems it brings along. ${ }^{10}$ Since Parsons uses the hyphen ('-') as his symbol for null assignment, we may assume that he does indeed work with Klima's zero or null element, even if he does not say so. Given the typographical oddness of the hyphen notation, I will go on using $\varnothing$ for the null element. But let us follow Parsons and see where it takes us.

Given his formal system, (4) is a proposition, where $F$ is the restrictor predicate and G the matrix predicate:

(4) (some $F x$ ) not (some $G y) x=y$

(for some $x$ that is $F$, it not so that for some $y$ that is $G, x=y$, or: Some $F$ is not $G$ )

Now, according to Parsons, when $\llbracket F \rrbracket=\varnothing$, so that, in Parsons' phrase, 'nothing' is assigned to the variable $x$ (though, in fact, $\boldsymbol{\sigma}$ is assigned to $x$ ), the part (some $G y$ ) $x=y$ is false for any value $\mathbf{e} \in \llbracket G \rrbracket$ for the variable $y$, simply because $x$ has received the value $\boldsymbol{\varnothing}$. More precisely, since the existential quantifier some is defined, for cases where $\llbracket F \rrbracket=\varnothing$, as requiring for truth that the scope of (some $G y$ ) - in this case $x=y$-is true with $\mathbf{e}$ for $\mathrm{y}$ and $\varnothing$ for $\mathrm{x}$, and since $\mathrm{x}=\mathrm{y}$ is always false when $\varnothing$ stands for either $\mathrm{x}$ or $\mathrm{y}$, it follows that $\boldsymbol{\sigma}=\mathrm{y}$ is false for any $\mathbf{e} \in \llbracket \mathrm{G} \rrbracket$ substituted for $\mathrm{y}$, so that (some $\mathrm{G} y$ ) $\boldsymbol{\sigma}=\mathrm{y}$ is false. Since (some $\mathrm{G}$ y) $\boldsymbol{\sigma}=\mathrm{y}$ is preceded by the negation, the part not (some $\mathrm{G} y$ ) $\boldsymbol{\sigma}=\mathrm{y}$ is true. The same procedure is now repeated for (some $F \mathbf{x}$ ): since the scope of (some $F \mathbf{x}$ ) - in this case not (some G y) $\boldsymbol{\sigma}=\mathrm{y}$-is true, the whole sentence (4) is true, even though $\llbracket F \rrbracket=\varnothing$.

Curiously, Parsons gives double definitions for the quantifiers, one for cases where the extension of the restrictor predicate is nonnull and one for cases where it is null. The universal quantifier every is defined as requiring for truth either, when $\llbracket F \rrbracket \neq \varnothing$, that every substitution for the variable bound by it delivers truth or, when $\llbracket F \rrbracket=\varnothing$, that its scope turns out true. Analogously, the existential quantifier some is defined as requiring for truth either, when $\llbracket F \rrbracket \neq \varnothing$, that at least one substitution for the variable bound by it delivers truth or, when $\llbracket F \rrbracket=\varnothing$, that its scope turns out true.

However, the distinction between cases where $\llbracket F \rrbracket \neq \varnothing$ and cases where $\llbracket F \rrbracket=\varnothing$ vanishes when it is admitted that null assignment amounts to the substitution of the null element $\varnothing$. Then EVERY can be defined uniformly as requiring that every admissible substitution for the variable bound by it delivers truth, and SOME as requiring that at least one admissible substitution delivers truth. This shows better than anything else that, as long as one stays within the bounds of a regular ontology without $\varnothing$, it is not possible, in Parsons' system, to provide the quantifiers with single uniform definitions respecting the MODULO-*-PRINCIPLE formulated above. But with the introduction of the ontologically vicious element $\boldsymbol{\varnothing}$, uniform definitions become possible. LOCA is, therefore, spoiled by its ontology.

But does it save the Square? My answer is that it does not. According to Aristotle (Prior Analytics $25^{\text {a }} 8-14$ ), $\mathbf{I}$ and $\mathbf{I}$ ! (Some $\mathbf{G}$ is $\mathbf{F}$-the inversion of $\mathbf{I}$ ) are equivalent. That this is so, is easily seen: $I$ is true just in case $\llbracket F \rrbracket \cap \llbracket G \rrbracket \neq \varnothing$; I! is true just in case $\llbracket G \rrbracket \cap \llbracket F \rrbracket \neq \varnothing$;

\footnotetext{
${ }^{10}$ Parsons seems to make use of the ploy used by Odysseus in Homer's Odyssey, when he escaped from the Cyclops Polyphemus, whose single eye he had just pierced with a red-hot club, by telling the Cyclops that his name was 'No-one'. When the blinded Polyphemus called on his fellow Cyclopes for help and revenge, he told them that No-one had pierced his eye, which made the other Cyclopes think that Polyphemus had been punished by the gods. In an epilogue called 'epicycle', Parsons discusses the philosophical question of the reification of 'nothing', quoting various ancient, medieval and modern authors. But he does not come to any clear conclusion.
} 
$\llbracket G \rrbracket \cap \llbracket F \rrbracket=\llbracket F \rrbracket \cap \llbracket G \rrbracket$; hence, I! is true just in case $\llbracket F \rrbracket \cap \llbracket G \rrbracket \neq \varnothing$. Likewise for the equivalence of $I^{*}$ (Some $F$ is not-G) and $I *$ ! (Some not-G is $F$ ): I* is true just in case $\llbracket F \rrbracket \cap \llbracket G \rrbracket \neq \varnothing ; I^{*} !$ is true just in case $\llbracket \overline{\mathrm{G}} \rrbracket \cap \llbracket F \rrbracket \neq \varnothing ; \llbracket F \rrbracket \cap \llbracket \bar{G} \rrbracket=\llbracket \bar{G} \rrbracket \cap \llbracket F \rrbracket ;$ hence, $I^{*}$ ! is true just in case $\llbracket F \rrbracket \cap \llbracket G \rrbracket \neq \varnothing$. In Aristotelian-Boethian and Aristotelian-Abelardian logic, as well as in standard modern logic, all of which have a symmetrical existential quantifier, both I and I* are thus equivalent with their inversions I! and I*!, respectively. In these logics, the following principle holds:

- Under the existential quantifier, the restrictor term and the matrix term (range and argument) are freely interchangeable salva veritate.

In the ø-variant of LOCA, however, which is claimed by its authors to be the proper analysis of the Square, this does not hold-unless the function $\neg \mathrm{G}(\mathrm{x})$ is (arbitrarily) banned and only lexically negative predicates like non-G(x) are allowed. This is shown as follows. Take a situation $\Sigma$ where $\llbracket F \rrbracket=\{a, b, c\}(a, b, c \in E N T)$ and $\llbracket G \rrbracket=\varnothing$. Some $F$ is not $\mathrm{G}\left(\mathbf{I}^{*}\right)$, analyzed as $\exists \mathrm{x}: \mathrm{F}(\mathrm{x}) \mid \neg \mathrm{G}(\mathrm{x})$, is true in $\Sigma$, since $\mathrm{a}, \mathrm{b}$ and $\mathrm{c}$ are the legitimate substitutions for $x$ in $G(x)$ and $\neg G(a), \neg G(b)$ and $\neg G(c)$ are all true, which satisfies the truth condition for $I^{*}$ as formulated above. However, Some not-G is $F\left(I^{*} !\right)$, analyzed as $\exists x: \neg G(x) \mid F(x)$, is false in $\Sigma$, since, given that $\llbracket G \rrbracket=\varnothing$, the only legitimate substitution for $x$ in $F(x)$ is the zero element $\varnothing$ yielding falsity for $F(x)$. LOCA thus not only fails to save the Square, it also undermines the set-theoretic foundations of the entire system.

One could propose to modify the substitution conditions for $\varnothing$ in such a way that all elements $\mathbf{e}$ in ENT are admitted in $G(x)$, since all $\mathbf{e}$ in ENT satisfy the range $\neg \mathrm{G}(\mathbf{x})$, which would make the range of the formula $\exists x: \neg G(x) \mid F(x)$ equal to ENT. This would make $I^{*}$ ! true, just like $I^{*}$. But now the problem reappears for a situation $\Sigma^{\prime}$, in which, again, $\llbracket F \rrbracket=\{a, b, c\}$ but $\llbracket G \rrbracket=E N T$. Now $I^{*}$ is false but $I^{*} !$ is undecidable since now all elements in ENT yield falsity for the range $\neg G(x)$ of $\exists x: \neg G(x) \mid F(x)$, which leaves this formula without any legitimate substitution for $x$ in $F(x)$ and hence with its truth value undecided.

Despite the contortions of Parsons' formal system, it is clear that, in the end, it reduces again to the simple system of restricted quantification enriched with the zero element as shown above in the examples (2) and (3). And this system is, in the end, untenable for the reasons given. We will, therefore, not regard LOCA as a viable avenue of research. It is a typical example of a logic that works in a purely technical sense but is vitiated by a paradoxical and vicious ontological element which makes the system run into logical aporia.

If the Square is to be saved - and this is indeed a worthy ambition-a totally different approach is to be followed, involving presupposition theory combined with an admission of virtual entities into the ontology and of a third truth value (radical falsity) into the logic. ${ }^{11}$ This requires no zero element and no restricted quantification, and it steers clear of the crass violation of natural intuitions perpetrated in LOCA. The Square is fully saved as a default bivalent logical system for those situations where all presuppositions of the sentences processed in the logic are fulfilled. The situations not fulfilling presuppositional truth conditions are then covered by a second, radical, negation which requires special

\footnotetext{
${ }^{11}$ This is not the place to elaborate the presuppositional solution to UEI. For an up-to-date and full discussion, see Seuren ([8]: Ch. 10).
} 
emphasis and a special intonation contour for the sentence as a whole, restoring truth for sentences suffering from presupposition failure.

The question is thus: what price is one prepared to pay for saving the Square? The choice is between, on the one hand, the introduction of an ontologically and settheoretically untenable null element plus a strident violation of natural intuitions and, on the other, the admission of virtual entities into the ontology and of two kinds of falsity into the logic, in strict adherence to natural intuitions. The former may deserve a place in abstract logical space, but it is sterile and without any wider interest; the latter produces a logic that is organically embedded in the human ecology of language, mind and world and helps explaining crucial facts of language and cognition. It is clear where our preferences should lie.

\section{References}

1. Ashworth, E.J.: Existential assumptions in late medieval logic. Am. Philos. Q. 10(2), 141-147 (1973)

2. King, P.: William of Ockham: Summa Logicae. In: Shand, J. (ed.) Central Works of Philosophy, vol. I, pp. 242-269. Acumen, Chesham (2005)

3. Klima, G.: Ars Artium. Essays in Philosophical Semantics, Medieval and Modern. Institute of Philosophy, Hungarian Academy of Sciences, Budapest (1988)

4. Moody, E.A.: Truth and Consequence in Mediæval Logic. North-Holland, Amsterdam (1953)

5. Parsons, T.: The traditional Square of Opposition. In: Zalta, E.N. (ed.) The Stanford Encyclopedia of Philosophy (October 1, 2006 revision). http://plato.standford.edu/entries/square/

6. Parsons, T.: Things that are right with the traditional Square of Opposition. Logica Univers. 2, 3-11 (2008)

7. Russell, B.: On denoting. Mind 14, 479-493 (1905)

8. Seuren, P.A.M.: The Logic of Language (= vol. II of Language from Within). Oxford University Press, Oxford (2010)

9. Seuren, P.A.M.: From Whorf to Montague. Oxford University Press, Oxford (to appear)

10. Thompson, M.: On Aristotle's Square of Opposition. Philos. Rev. 62(2), 251-265 (1953)

P.A.M. Seuren $(\bowtie)$

Max Planck Institute for Psycholinguistics, PO Box 310, 6500 AH Nijmegen, The Netherlands e-mail: pieter.seuren@mpi.nl 